\title{
A Study of Bio-Computational Design in Terms of Enhancing Water Absorption by Method of Bionics Within the Architectural Fields
}

\author{
Gang $\mathrm{Mao}^{(\otimes)}$ \\ The Bartlett School of Architecture, University College London, 22 Gordon Street, London, UK \\ g.mao@ucl.ac.uk
}

\begin{abstract}
This essay aims to explore an architecture computational design intended to accept and absorb moisture through geometrical and material conditions, and using design strategies, help deliver this moisture upwards through capillary action to areas of cryptogamic growth including mosses and smaller ferns on the surface of architecture. The purpose of this research project is to explore the morphology of general capillary systems based on research into the principle of xylematic structures in trees, thereby creating a range of capillary designs using three types of material: plaster, 3D print plastic, and concrete. In addition, computational studies are used to examine various types of computational designs of organic structures, such as columns, driven by physical and environmental conditions such as sunshine, shade, tides and other biological processes to explore three-dimensional particle-based branching systems that define both structural and water delivery paths.
\end{abstract}

Keywords: Bio-computational design · Bionics · Bio-receptive design ·

Xylematic structure $\cdot$ Parametric design

\section{Bio-Receptive and Bio-Colonization}

In ecology, the growth of epiphytes on tree bark is scenery that can commonly be seen practically everywhere. This is an example of what is known as bio-colonisation (Cruz and Beckett 2016). Figure 1 illustrates the most typical examples of this natural phenomenon.

These photographs illuminate different trends of green growth on tree bark, showing the importance of direction and the effects of surrounding micro-climatic conditions (Woodell 1979). The growth never encompasses the entire circumference of the tree and also changes with the season. Moisture is a crucial factor in this. Plants tend to grow on the branches and at the base of the host plant. One possible reasons for this is that tree trunks and branches tend to have a greater capacity to hold moisture than the other plant parts (Honda 1971). These two photographs show how important water is for growth. A tree can be considered to consist of two distinct parts, each with different degrees of moisture: the tree itself and the transition zone, which is a bio-receptive part defined by the amount of water. 

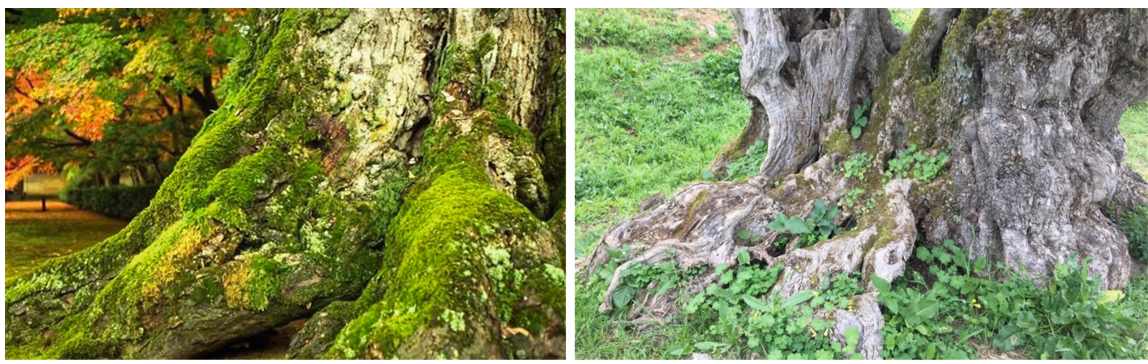

Fig. 1. (Left): Common view in London (Right): A tree colonized by micro-plants (Author)

Water has been identified as the critical defining factor in terms of the subsequent area where growth occurs (Wen et al. 2019); this phenomenon can also be seen in relation to architecture, as shown in Fig. 2, which depicts moss coverage on the vertical surface of a building. The notable element here is the fact that the moss is not growing everywhere. This results from water dripping down the wall, which slightly saturates the rock, providing suitable conditions for plant growth. There is also a transition zone seen on building surfaces, not only from plants to the building but also from nature to architecture.

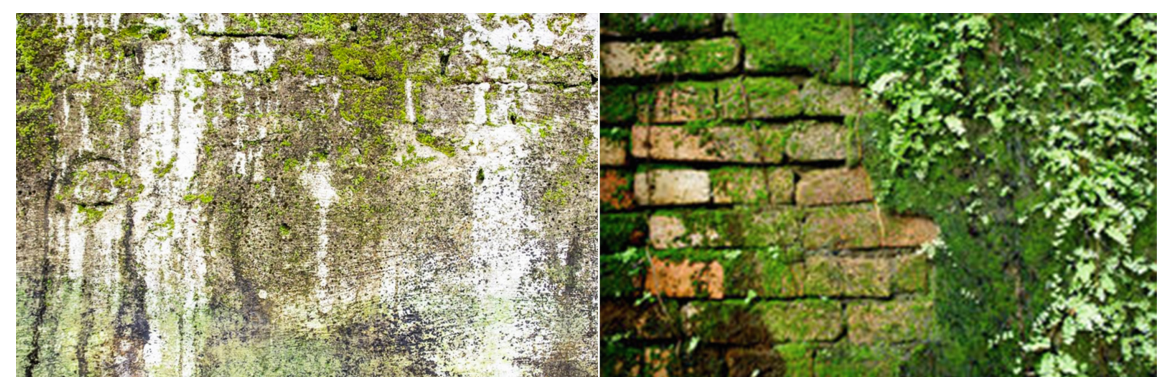

Fig. 2. (Left): Common view in London (Right): A tree colonized by micro-plants (Author)

This transition zone and the relationship between these two elements are of particular interest. In fact, the transition zone can be seen as more of a link between architecture and water, since the water can define the area of vegetative growth (Wen et al. 2019). This development is not only created by nature; human beings also attempt to use this method to control the relationship between nature and buildings.

Taking the city of Suzhou Fig. 3 as an example; a number of traditional buildings have either directly or indirectly sunk into the water. Historically, this was a common design strategy in traditional Chinese architecture so as to deal with the link between water and buildings. Many building materials provide an agreeable environment for the growth of vegetation such as moss and algae. With these kinds of plants growing on the surface, a transition zone can occur. 


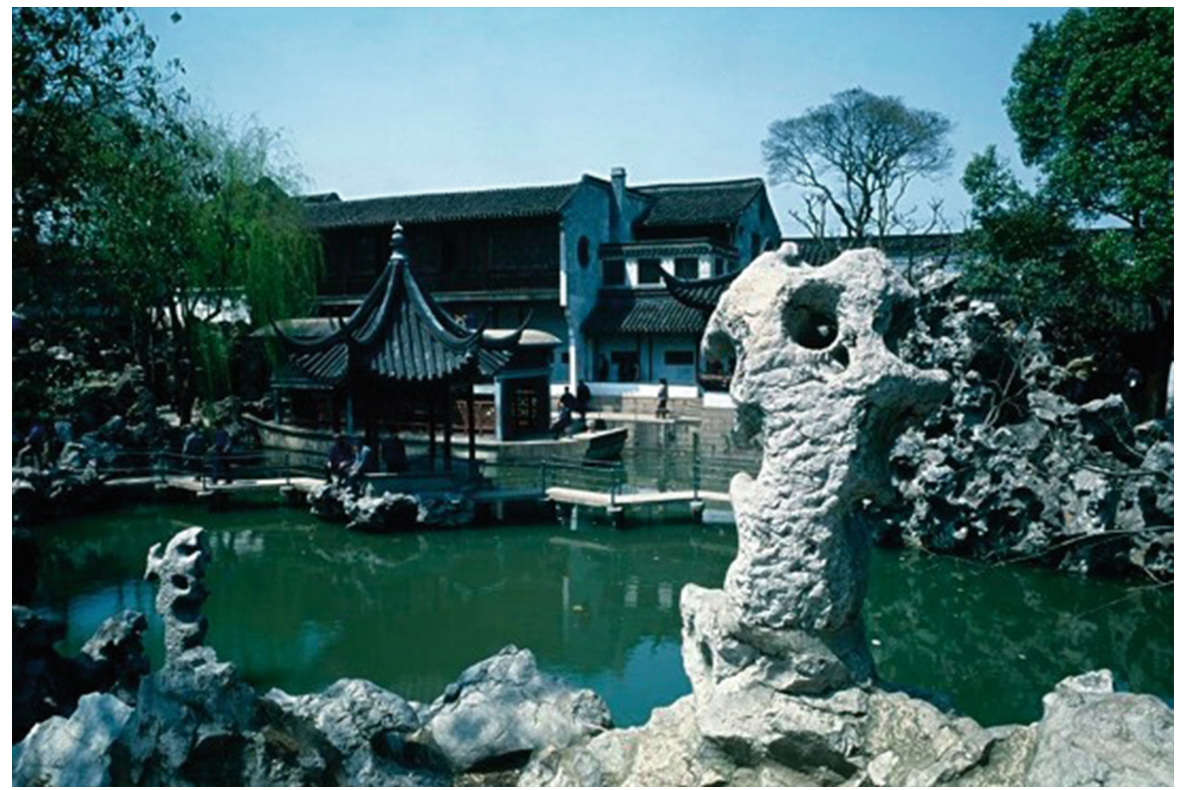

Fig. 3. Garden in Suzhou (Liuhe Travel 2017)

\section{The Introduction of Transition Zone}

The aforementioned examples show that designers in the past tended to form a natural link between architecture and nature. This link can be defined as a transition zone. In addition, as previously mentioned, the transition zone is a place where building materials allow for easy vegetation growth on its surface. The junction area in architecture should also be mentioned. The absorption of water by the surfaces of buildings subsequently creates an environment conducive to the growth of plants. This means that buildings and water are not isolated from each other, as they have a transition zone between them. The transition zone between the two elements can be regarded as a man-made place (Cruz and Beckett 2016), which provides a habitat for plants to grow in; in other words, this zone makes the building surface bio-receptive (Cruz and Beckett 2016). It is worth paying attention to the transition zone, as it is essential for the benefit of our environment.

While undertaking this project, focus will be placed on computational designs that can be used to enhance water absorption (extra water is not useful for the building but can still be absorbed by the building material) which makes it bio-receptive, thus in turn encouraging plants like moss or algae that can benefit the environment to grow. Building materials can be designed to deliberately absorb water and encourage chosen vegetation to grow on the surface. Therefore, it is feasible for architecture to be designed in a way that encourages the growth of green walls, also known as vegetated walls, to control heat. The temperature of the ambient air, interior air, and exterior walls of buildings can be effectively reduced through the use of a green façade (Jeffrey W. 2010). This has led to the idea of an initial design concept that encourages the absorption of more water to encourage growth of vegetation. 
The project presented here is based on the following two points:

1. Bio-mimetic principles: through simulation of the complex structures produced by nature, which is the principle of the xylem system, different prototype forms can be obtained.

2. Computational design: based on the principles learned from nature, integrated algorithmic logic can be applied in the design process, allowing designers to create a complex set of constraints.

With these points in mind, it is important to gain a basic understanding of how water absorption occurs in nature.

\section{Water Delivery Path in Nature}

As previously mentioned, it is essential to understand the basic principles of water delivery as they exist in nature. First, trees are to be considered, as trees are generally the existing tallest plants, and they can obtain water from both the air and soil. Despite the fact that many trees have a height in excess of ten meters, it is still possible for water to be successfully transported from the tree root system directly to the highest point of the tree (Susman et al. 2011). Such water transportation ability found in trees provides an initial reference for further research into this phenomenon, so as to fully understand the capacity of trees to effectively move water in such a manner. The main reasons for this, which will be explored in more detail, are osmosis, transpiration, and capillary action. As Susman said in 2011, “... 'pumping activity' originating in the 'life form' for the upward flow of water is not necessary...", and thus other less understood mechanisms will not be considered in this work.

The first factor that influences water absorption is capillary action. This is the most common answer to the question of how water can be transported to the top of trees, and indeed, capillary action is one of the mechanisms that enables this upward flow. However, it is not sufficient in itself. Water channels are also used to transport water across membranes. These channels are believed to be involved in many physiological processes, including water transportation in trees. Cohesion, adhesion, and surface tension combine to create capillary action. While some energy is consumed using this method, it supplies a significant source of moisture for vegetation without a large outlay of such energy (Thomas 2015).

There are two types of transport tissues found in plants. Water is transported through a complex tube system that is formed by hollow dead cells named xylem. The main function of xylem is to transport water from the roots within damp soil to the crowns of trees. The phloem transports nutrition from the leaves to the rest of the tree. The water-transportation cells found in mature xylem are all dead, which highlights the fact that the transport of water is an essentially passive process with only a minuscule active root pressure component. The function of the 'hairs' on the roots is to absorb water that can then be transported via the xylem vessels to the crown. A further phenomenon which facilitates this movement of water is transpiration, process in which water evaporates from the leaves of the tree (Fig. 4). 


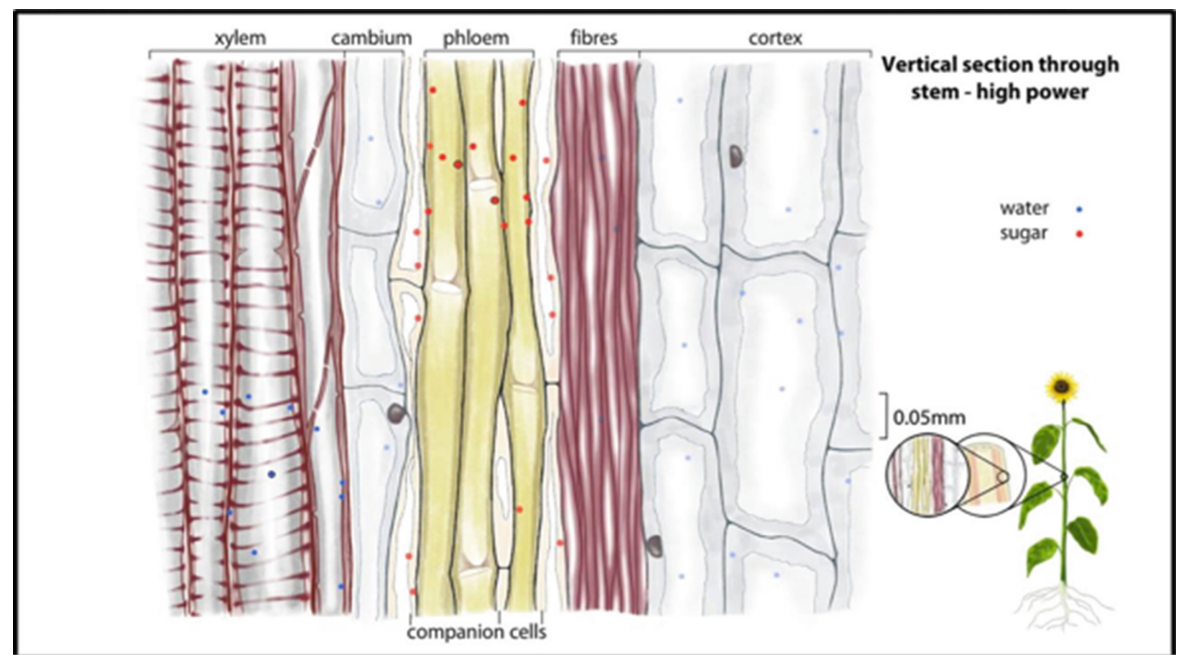

Fig. 4. Vertical section through tree (Chahhla Sadek 2015)

\section{Computational Design Principle}

Based on the results gathered from research conducted into the tree, an initial design method can be created and computational methods can be used to design construction techniques through use of appropriate technology. Architects use a varying range of different design strategies, but this type of computational design is becoming a common trend in the design field, and is described as an efficient way to gather and calculate information (Menges 2012). With the assistance of a computational model, it is possible for architects to forecast the form of any other alternative design way (Menges and Ahlquist 2011). Turing, who is known for his work in computing, but was also a biologist and mathematician, was one of the most important people in the field of computational generation. In his book, Morphogen Theory of Phyllotaxis, he explains the process of using geometrical descriptions of patterns. He also implemented mathematical equations to gain an understanding of the reaction-diffusion effect, and a chemical model to solve the equations for small perturbations.

This current project makes use of the methodology used by Turing in his study of phyllotaxis, effectively tackling the problem in two stages. The first stage is an attempt to analyze the structure of plants; following this, a computational design is created to explain the prototype.

The concept of this project originates from the theory of Water Transport in Plants as a Catenary Process, written by Van Den Honert, T., in 1948. In this book the author indicates that the transportation of moisture through roots, xylem and leaves may be considered to be a catenary process. Thus, the initial particle-based branching system concept can serve as the biological design. An important feature of the first stage is examination of the structure of xylem based on the vital role it plays in water transportation. The xylem system works on a particle system principle, as the xylem system is formed by many dead cells. Therefore, water is transported to the top through means of 
differences in density. Simulation of this crucial particle system was conducted during this project through use of the Houdini modelling software.

Xylem vessels are combined with dead cells, with the structure of xylem as a tube system with a range of tissues, as introduced by Turing in his theory. A large number of particles can be connected in order to create such a tube, and several tubes can be combined so as to form a xylem system. The impulse count reflects the number of original particles, and different impulse counts create different forms. This concept can be seen in Fig. 5, which illustrates the two- and three-dimensional results obtained from use of various quantities of original particles.
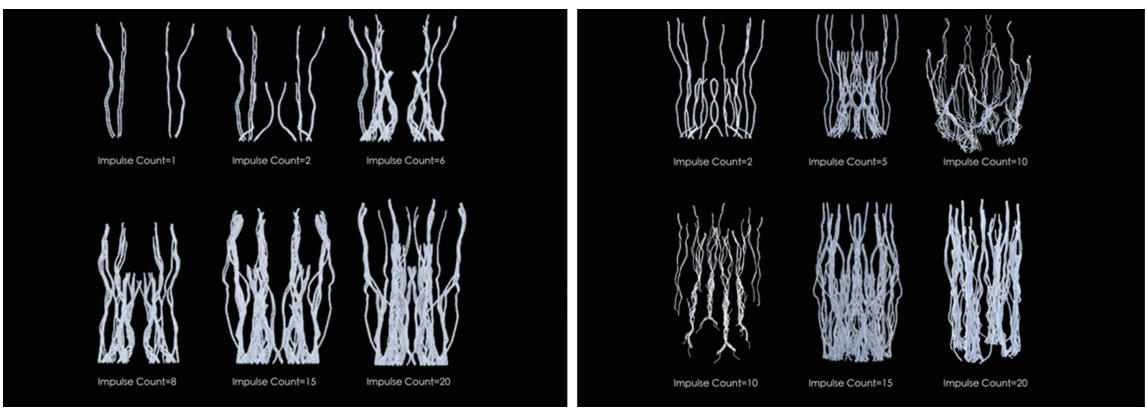

Fig. 5. (Left): 2D branching system (Author) (Right): 3D branching system (Author)

The two-dimensional form can be used to mimic the results of a xylem system, however, if the particles are modelled in a three-dimensional space, the resulting outcome will be different. This is just like emergence: where a large archetype appears through interactions by smaller and simpler entities, causing the larger archetype to exhibit properties that the smaller and simpler archetype does not show. Particles are the small simple entities. When a trail follows the growing particle the smaller piece will disappear and a new piece will appear. For example, the first particle is used and upward motion is applied to it, thereby simulating a natural growth pattern; subsequently, the next generation of particles will follow this upward motion. If both are kept in this design, the result is a large piece and even though the small particle will not be apparent, but is still there. This is similar to a collective intelligence, using the emergence phenomenon to create a new piece.

Once the basic simulation system has been tested by mimicking the xylem form, further research is required so as to enable the generation of more complicated designs. As the xylem tube system has a horizontal component based on branching systems, research into branching systems is therefore required. Though utilization of Turing's method, this analysis can be simplified and adapted to the investigation of several different kinds of trees in the UK, which will in turn lead to an understanding of the common features of such trees. After completing relevant analysis, results point to the fact that there are fewer branches on the northern side of most trees in comparison to the southern side. Moreover, moss is most likely to grow on the shady side of a tree. Branching systems can play a significant role in the growth of trees. One key point is the growth method at the fork of branches, which represents the major growth factor. This is because the way 
in which each branch grows is decided by the position of the fork and the amount by which a twig is separated from the fork. This growth can be mathematically determined using the system developed by Aristid Lindenmayer in 1968.

For the purpose of the simulations conducted as part of this study, Lindenmayer's system, also referred to as the 'L-system' was employed. The L-system is both a parallel rewriting system and a type of formal grammar. It is thus necessary to understand how to use digital language to rewrite generated strings and translate these into geometric structures.

Basic plant models and natural-looking organic forms are relatively easy to define, however, if the recursion level is increased, the form will slowly grow and become more complex. This recursive principle is shown below in Fig. 6:

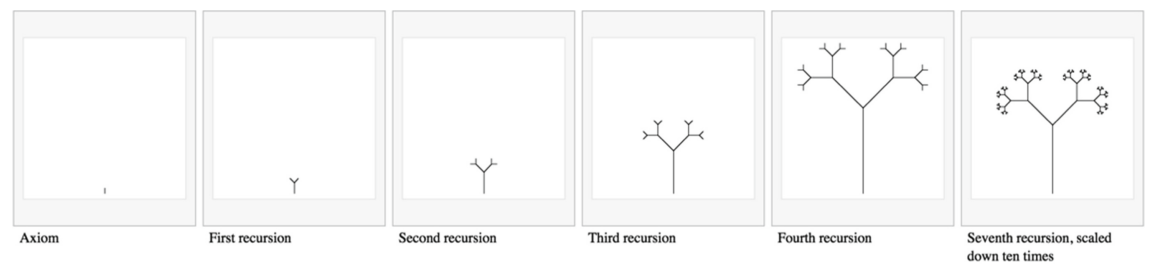

Fig. 6. Growth method of the L-System

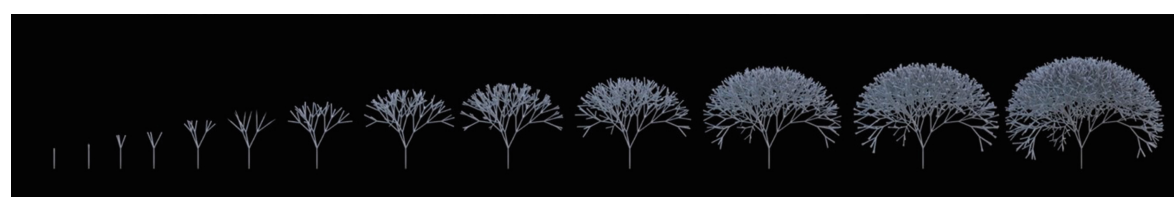

Fig. 7. Growth method of the L-System in Houdini (Author)

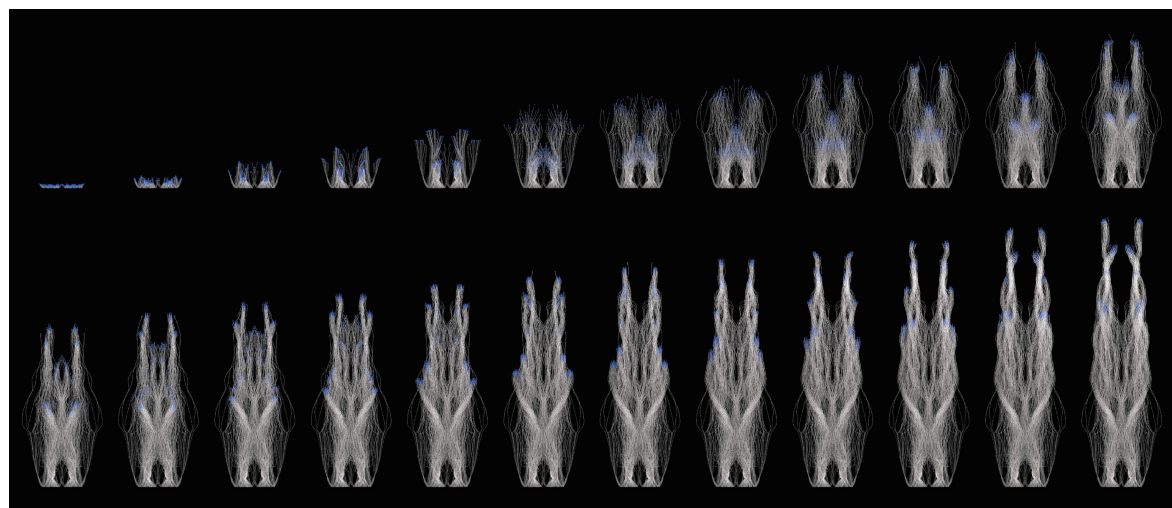

Fig. 8. Growth method of the column shape-based L-System in Houdini (Author)

The Houdini software was chosen to simulate this L-system growth process. The first generation is a single pillar, and subsequently, the pillar splits at the top from the 
next generation. Repetition of this process results in simulated growth of a tree, as illustrated in Fig. 7. This effectively replicates the fundamental growth principle as is found in nature. The simulation of this natural growth process is a means of developing the design required for the purpose of this project, therefore, the growth method of the column shape-based L-System in the Houdini software has also been tested, as seen in Fig. 8.

In terms of design, it seems unreasonable to merely mimic a principle from nature. It would be a shame to merely design an artificial tree or some other plant for our world. Simulating the principle of the L-system is something worth doing at the beginning of the design process. As the process shows below, 'growth' starts with a grid, then it begins to split and have an upward motion which represents phototropism in plants. After five generations, numerous points in this object start to split, creating a branching system at the end of the process. This is one example of how the L-system principal is simulated through use of upward motion. However, roots in nature also use this principle in downward motion, so it is necessary to also design a downward motion derived structure. Figure 9 and Fig. 10 illustrate the results obtained; the natural root system performs two functions, anchoring the structure in the soil and also absorbing moisture from the soil. Both of these features can be incorporated into the design for this project.

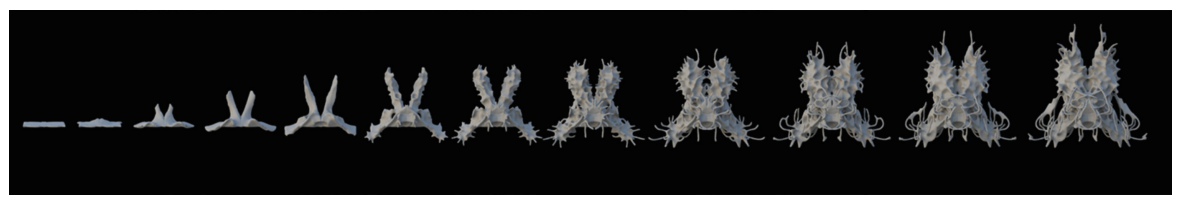

Fig. 9. Design simulation of L-system with upward motion (Author)

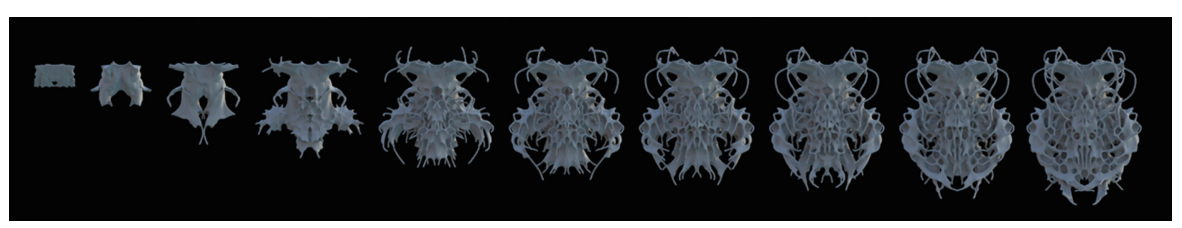

Fig. 10. Design simulation of L-system with downward motion (Author)

The design simulation of the L-system is shown in Fig. 9 and Fig. 10. At first, only one grid exists. After applying an upward motion, the grid starts to split. This is similar to the behavior of the particle in the basic L-system principle, however, does not simply mimic the process of the L-system, as the split points do not occur just on the top of the model, but can occur almost everywhere. It is important to note that growth can occur in both upward and downward directions.

\section{Bionics Within the Architectural Fields}

In general, this simulation clearly shows the mathematical principles of tree growth, providing a theoretical basis for the establishment of a tree model. However, the difference 
between this case and the project as a whole is that the project is based on the natural principle of biological simulation, with a purpose to create a new structure in which certain aspects are based on simulated branch shapes rather than simply copying natural branch forms. As the intention is to formulate a new scenario for the transition zone (i.e., the place where architecture transitions to water), many possible design variants may exist. Some possibilities have been explored, including urban furniture (Fig. 11) and different sizes of columns (Fig. 12). Assigning different numbers of points and different particle sizes in Houdini, results in a variety of designs can be generated, as shown below.

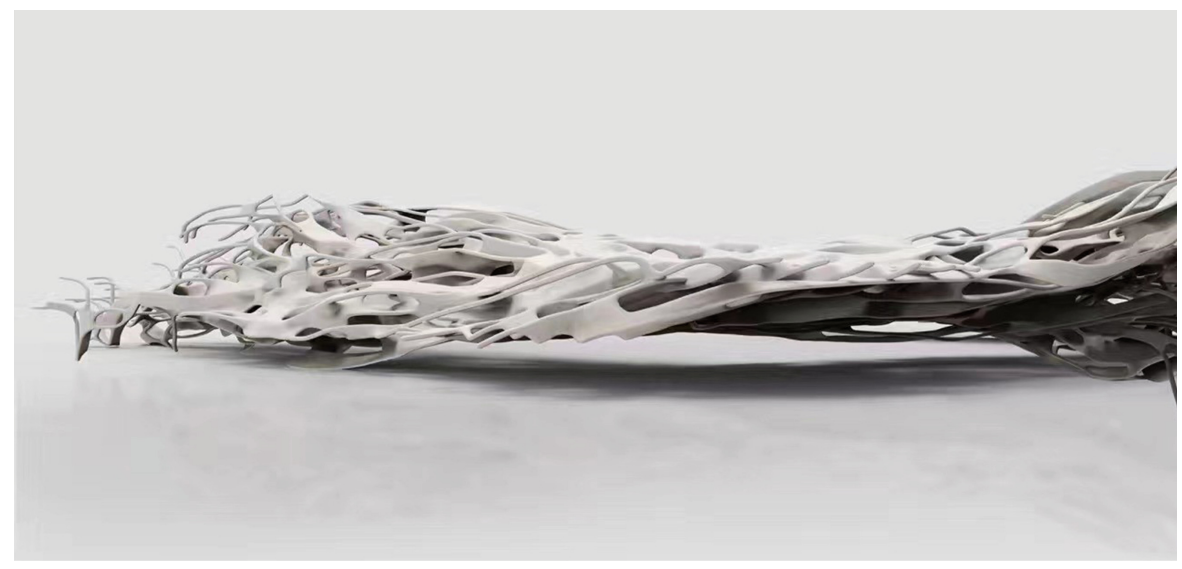

Fig. 11. Designs of different urban furniture (Author)

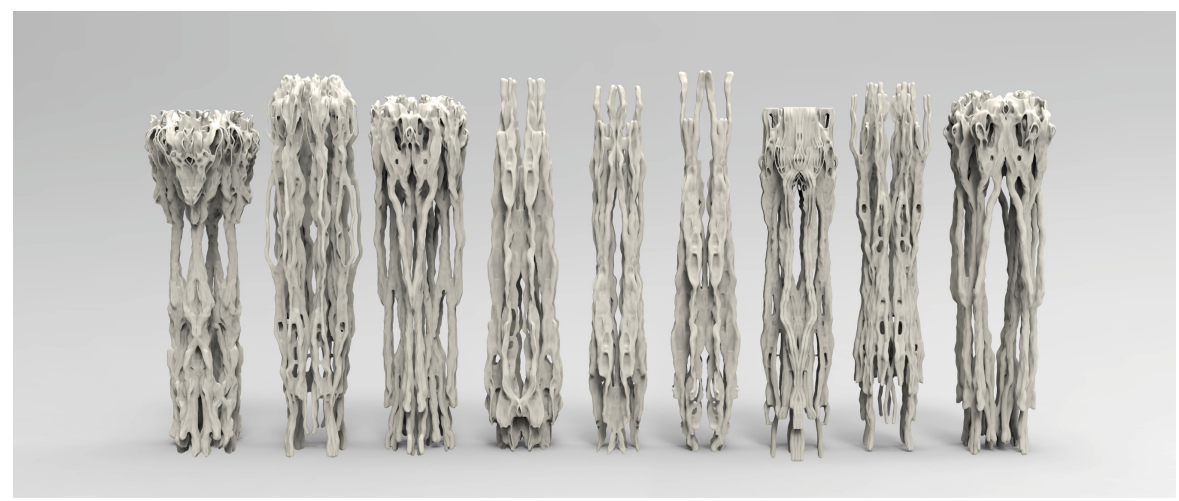

Fig. 12. Designs of different urban columns (Author)

\section{Fabrication Method}

\subsection{Material Test}

A variety of tests were conducted in order to determine the optimal manner in which to control the movement of water from the bottom of the structure to the top. The testing of 
various materials played a helping hand in determining the most appropriate materials for the design. One of the tests conducted related to ease of fabrication of the structure. Plaster was a prime candidate, as an easy to acquire building material which can be quickly formed into shapes. Thus, it was considered to be a potential material for use in this project. However, it is not an ideal material for the structure since it easily absorbs water, making it fragile. Based on this, the next step was to consider MPC concrete. This is a porous material that is beneficial for vegetation growth. The material tests conducted using MPC concrete revealed that it has the capability to contain water, but also revealed that this ability does not depend on the size of the aggregate. After many material tests, a small sized aggregate was chosen as one of the materials for this project, as it can be used as a structure system. From examining these two materials, it can be seen that each has advantages and disadvantages. While plaster is capable of absorbing more water, it tends to be quite fragile. Conversely, MPC concrete is very bio-receptive but it is not an ideal material for absorbing water. The solution to this problem is the introduction of an additional layer consisting of 3D printed plastic. This layer not only serves as a structural layer but also provides the model with an elegant geometric element. Therefore, it is determined that this proposed combination of construction materials would have the greatest probability of delivering an optimal outcome.

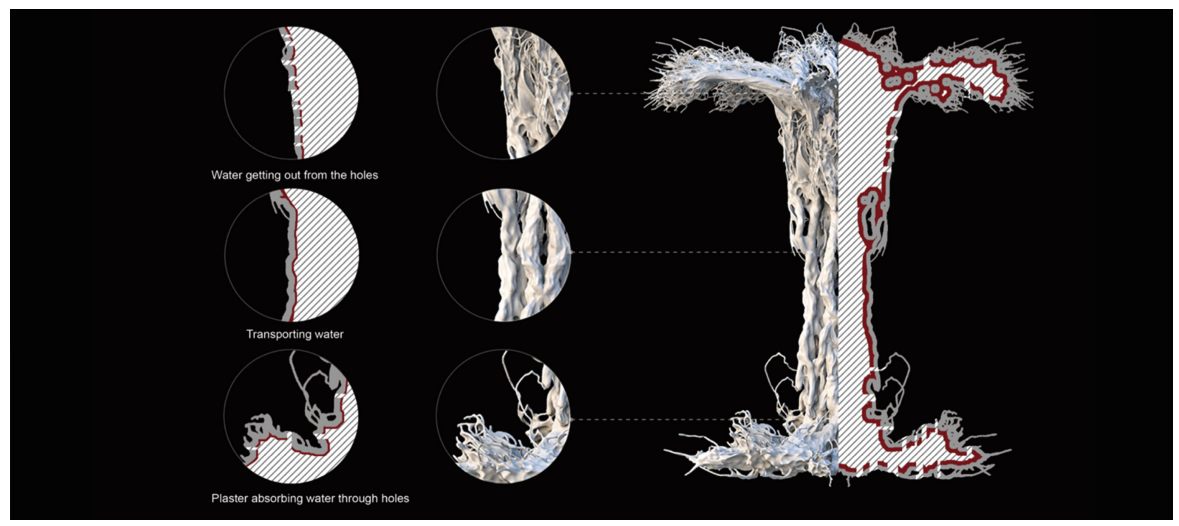

Fig. 13. (Left): Three layers of materials. (Middle): Details of the structure. (Right): Left-side is the design, right-side is the section (Author)

\subsection{Fabrication Test}

As shown in Fig. 13, the model consists of three layers. The inner layer will be filled with plaster that can absorb water; the second layer will be a 3D print layer, providing geometry and helping with growth; and the third layer will be concrete, forming a structural layer. However, use of these three models still poses a problem as the 3D print model may prevent the water from coming out, making the entire project redundant.

The solution to this is the inclusion of several holes in the model that would allow water can come out from the inner plaster, thus allowing plant growth. This is also a 
method which grants the designer the ability to decide the exact locations in which the growth of plants will be permitted. It provides more flexibility because different conditions exist in different environments, meaning that growth will not always happen in the same place. The use of these holes will allow the quantity of water that is delivered to be adjusted accordingly, thus controlling the precise area of growth. Finally, the results obtained from creating these branching systems will be combined with the inner extraction plaster system and the bio-receptive architecture bark system. In relation to the fabrication method, the idea of spraying concrete is under consideration.

\section{Conclusion}

This project aims to design and fabricate an organic and flexible construction through simulation of the growth processes of natural plants, with particular focus on their capillary systems, based on particle-based growth for a transition zone. The design result is thus decided by environmental conditions. The basis for this work is provided by an analysis of xylem and capillary systems, with the design being completed through creation of digital models of these two systems based on the conclusion of the analysis. The proposed outcome of this project is the creation of a bio-receptive scaffold that can absorb water from the soil and provide a suitable habitat for micro plants to colonize. The proposed design for this project is specifically tailored to the transition zone. Despite not being a true plant, it does employ a plant-inspired basis of water delivery. In addition, although the structure does not follow conventional architectural protocols, it uses building materials that are beneficial for the environment. It could, therefore, be considered to represent a transition design between nature and architecture, similar to the origin of the design concept - the transition zone.

\section{References}

Turing, A., Saunders, P.: Morphogenesis, 1st edn. North-Holland, Amsterdam (1992)

Cruz, M., Beckett, R.: Bioreceptive design: a novel approach to biodigital materiality. Archit. Res. Q. 20(01), 51-64 (2016)

Chiu, S., Ewers, F.: Xylem structure and water transport in a twiner, a scrambler, and a shrub of Lonicera (Caprifoliaceae). Trees 6(4) (1992). https://doi.org/10.1007/BF00224339

Susman, K., Razpet, N., Čepič, M.: Water transport in trees-an artificial laboratory tree. Phys. Educ. 46(3), 340-347 (2011)

Gadd, G.: Geomicrobiology of the built environment. Nat. Microbiol. 2(4), 16275 (2017)

Malik, F., Clement, R., Gethin, D., Krawszik, W., Parker, A.: Nature's moisture harvesters: a comparative review. Bioinsp. Biomimetics 9(3), 031002 (2014)

Guillitte, O.: Bioreceptivity: a new concept for building ecology studies. Sci. Total Environ. 167(1-3), 215-220 (1995)

Sui, H., Han, B., Lee, J., Walian, P., Jap, B.: Structural basis of water-specific transport through the AQP1 water channel. Nature 414(6866), 872-878 (2001)

Prieto Lamas, B., Rivas Brea, M., Silva Hermo, B.: Colonization by lichens of granite churches in Galicia (northwest Spain). Sci. Total Environ. 167(1-3), 343-351 (1995)

D'Orazio, M., et al.: Effects of water absorption and surface roughness on the bioreceptivity of ETICS compared to clay bricks. Build. Environ. 77, 20-28 (2014) 
Guillitte, O., Dreesen, R.: Laboratory chamber studies and petrographical analysis as bioreceptivity assessment tools of building materials. Sci. Total Environ. 167(1-3), 365-374 (1995)

McCulloh, K., Sperry, J., Adler, F.: Water transport in plants obeys Murray's law. Nature 421(6926), 939-942 (2003)

Woodell, S.R.J.: Phytosociology. Ecology 60(5), 1079-1080 (1979). https://doi.org/10.2307/193 6880

Honda, S., Hongladarom-Honda, T., Kwanyuen, P., Wildman, S.: Interpretations on chloroplast reproduction derived from correlations between cells and chloroplasts. Planta 97(1), 1-15 (1971)

Wen, Y., et al.: Cumulative effects of climatic factors on terrestrial vegetation growth. J. Geophys. Res. Biogeosci. 124(4), 789-806 (2019)

Open Access This chapter is licensed under the terms of the Creative Commons Attribution 4.0 International License (http://creativecommons.org/licenses/by/4.0/), which permits use, sharing, adaptation, distribution and reproduction in any medium or format, as long as you give appropriate credit to the original author(s) and the source, provide a link to the Creative Commons license and indicate if changes were made.

The images or other third party material in this chapter are included in the chapter's Creative Commons license, unless indicated otherwise in a credit line to the material. If material is not included in the chapter's Creative Commons license and your intended use is not permitted by statutory regulation or exceeds the permitted use, you will need to obtain permission directly from the copyright holder.

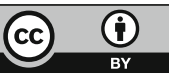

